\title{
ABSTRACTS OF INVITED AND SCIENTIFIC PAPERS
}

\begin{tabular}{|l|}
\hline Symposium-I \\
Quality Management of Emergency \\
Medical Services \\
Monday, 10 May, 13:00-15:00 hours \\
Chair:Jean Marie Fonrouge; Naruo Uehara \\
\hline
\end{tabular}

S1-1

An Overview of Current Approaches to Quality in Health Services

Naruo Uebara, $M D$

Professor, International Health, Tohoku University School of Medicine, Japan

"Quality Assurance and Improvement in Healthcare" currently is a worldwide trend, and various approaches are being undertaken toward enhancing the quality of emergency medical care and emergency medical service systems. In addition to the traditional approaches, such as Continuous Medical Education, Specialty Development of Emergency Physicians, and Accreditation of Emergency Departments, new approaches are being developed and tested. These approaches adopt the concept of KAIZEN or Total Quality Management (TQM) have been tested in many countries, and include: 1) Quality Control (QC) Circles; 2) Continuous Quality Improvement (CQI); 3) Evidence-Based Medicine; 4) Critical Pathways; 5) Practice Guidelines; 6) Customer Satisfaction Surveys; and 7) Performance Indicators.

The primary features of the concept of TQM are system-wide, process-oriented, patient-centered, and evidence-based. In TQM, "certainty" of care is of greater concern than is "excellence" of care. This is illustrated with the well-known catch-phrase of "failure is treasure land" (Failure cases provide us with invaluable improvement opportunity.")

After reviewing the original concept of TQM/KAIZEN in Japanese industries and the evolution of the concept of quality in health care in the USA, an overview of various quality approaches will be introduced and discussed with the aim of facilitating the following panel discussion.

Keywords: continuous quality improvement (CQI); health care; management; quality; quality assurance (QA); total quality management (TQM)

\section{S1-2}

Quality Improvement for Emergency Medicine in Australia

Peter Cameron

Associate Professor, Director of Emergency Medicine, Royal Melbourne Hospital, Melbourne, Australia

Historically, Australian hospitals have had extensive Quality Assurance programmes in an attempt to maintain standards. Like other industries, during the last 10 years, there has occurred the realisation that better processes developed when employees focused on quality improvement rather than maintaining standards. Now, in most hospitals, the useless accumulation of mountains of data purporting to measure quality has ceased. Quality Councils have been implemented with a view to identifying areas of concern, instituting changes, and measuring the effects of these changes. Strategies that appear to have resulted in improvements in Emergency Medicine performance include:

1) The Victorian Emergency Services Enhancement Program - Financial bonuses attached to reducing waiting time and admission delays have resulted in significant improvements;

2) The Australian Council of Hospital Standards (ACHS) has changed its focus from measuring "standards" to examining Quality Improvement (QI) processes, thus forcing hospitals to demonstrate working models of the QI process on inspection.

3) Round Table/Benchmarking Exercise - Hospitals now compare performance in key areas including emergency medicine and identify potential targets for improvement.

4) Systems of Care - Emphasis on continuity of care from prehospital to hospital and back to the community again. Emergency Medicine plays a key role in the interface between community and hospital.

Improvements in access to hospital care are dependent on the efficient use of hospital beds. Emergency Medicine plays a critical role in this triage process.

With respect to prehospital care, the focus still clearly is on improving response times, especially for cardiac arrest and trauma. There is a trend to greater medical control and audit. The value of many prehospital interventions is being questioned. A database with common elements is being developed at State and National levels. This should lead to better National and International benchmarking.

Keywords: beds; benchmarking; continuity; Emergency Medicine; hospitals; prehospital; quality assurance; quality improvement; standards

\section{S1-3}

Effects of Comprehensive Quality Management on Reduction of Emergency Patient Processing Time Dong Pill Lee

Department of Emergency Medicine, Kaemyung University School of Medicine

Objective: To determine whether the use of a quality assurance program in Emergency Medicine was able to shorten patients' waiting time in the Emergency Department (ED).

Method: Following a baseline study of the delay of patients in the ED, we developed serial goals of patientprocessing time, and analyzed the factors related to the process delay, conducting various meetings periodically with feedback of the results from each study and applied new management skills. The statistical methods used 
were ANOVA and LSD.

Results: During the period of June 94 through December 95 ( 1.5 years), the average ED patient processing time from registration to disposition, was reduced by about two hours $(p<0.05)$ as well as the reduction of variations among the different specialties (SD from 2.43 to 1.28 ).

Conclusion: Quality assurance programs in ED may be able to reduce patient waiting time to significant degrees.

Keywords: delays; emergency department; processing time; quality assurance; waiting time

\section{S1-4}

Quality Management of Emergency Medical Service in Japan

Kimitaka Tajimi, $M D ;{ }^{1}$ Hiroshi Kin, $M D ;^{2}$

Jun Takezawa, $M D^{3}$

1. Trauma and Critical Care Center, Teikyo University School of Medicine, Tokyo, Japan

2. Funabashi City Medical Center, Funabashi, Japan

3. Emergency Medicine/Intensive Care Unit, Nagoya University, Nagoya, Japan

The previous observational cohort study by Kohama, et al in 1990, indicated that the overall survival rate of out-ofhospital cardiac arrest (OHCA) in Japan was lower than that of the western, industrialized countries. Based on this report, Kyukyu-Kyumei-Shi (Emergency Medical Technician) system in Japan was established in 1991 in an effort to improve outcome from OHCA. However, recent investigations suggest that Kyukyu-Kyumei-Shi system did not improve overall mortality from OHCA.

Overall mortality (case mix) is not a good indicator of quality assessment of emergency medical service (EMS), because background etiology of OHCA influences outcome. Most of Japanese physicians consider that the incidence of cardiac etiology of OHCA is lower than is that in the western industrialized countries. However, this speculation can not be proven due to the lack of epidemiological data.

The Utstein style of reporting of data for OHCA allows us to provide more appropriate comparisons. Many studies based on Utstein style, emphasize that only patients with $\mathrm{OHCA}$ who are with ventricular fibrillation (VF) or ventricular tachycardia (VT) have a moderate prospect of survival. Therefore, it seems we should use the outcome of VF/VT as an indicator for quality assessment of EMS.

The survival rate (discharged alive) from witnessed VF/VT in Funabashi City was $18.1 \%$, almost the same as that of the western industrialized countries. However, other parts of Japan have a lower survival rate, even after Kyukyu-Kyumei-Shi was introduced. Funabashi City is the only city in Japan that has a two-tiered EMS. The future prospective of two-tiered EMS in Japan should be discussed.

The population-based incidence of witnessed VF/V'T due to cardiac etiology in Japan was 1.9 cases $/ 100,000$ population, which is one tenth of that in the western industrialized countries. It is estimated that there are only 2,000 VF/VT cases per year throughout Japan. In the meantime, 6,000 Kyukyu-Kyumei-Shi already have become involved in EMS. This matter needs to be reconsidered based on the cost-benefit analysis.

Although the need for implementation of continous quality improvement (CQI) for Japanese EMS has been discussed after establishing Kyukyu-Kyumei-Shi system, such a concept has not been well-realized as a tool for system evaluation.

Keywords: cardiac arrest; cost-benefit; emergency medical services (EMS); incidence; Kyukyu-Kyumei-Shi; out-of-hospital; prehospital; quality; tiered system; Utstein; ventricular fibrillation; ventricular tachycardia

\section{S1-5}

Current Initiatives on CQI of Emergency Medical Services in the United States

James Loftus

Department of Emergency Medicine at Cedars-Sinai Medical Center in Los Angeles, California USA

Objective: Can patient satisfaction scores be improved using continuous quality improvement (CQI) methodology?

Method: Like many emergency departments, we historically have prided ourselves on the quality of care given to our patients, but were suprised to discover that our patient satisfaction scores as measured by NCG, were lower than expected. This prompted us to embark upon a program of continuous quality improvement. In the first phase of the program we analyzed the results of the patient satisfaction survey to identify those areas that could be improved. The initial efforts included focus groups of patients in the community who use our service and meetings with all employees who worked in the Department. Hospital quality management experts worked with us to create a process to improve our overall scores. We also examined patient satisfaction scores for individual physicians, and developed a plan to improve these scores. Armed with tools learned at Institute for Healthcare Improvement Breakthrough Series, we sought to decrease waits and delays in our Department, which was a major component of patient dissatisfaction.

Results: The mean patient satisfaction score for the "Total Process" improved from $66 \%$ in Wave I to $76 \%$ in Wave XV, and physician specific scores increased from $67 \%$ to $78 \%$ during the same period time.

Conclusion: Patient satisfaction scores can be improved by using CQI methodology.

Keywords: continuous quality improvement (CQI); delays; emergency department; waiting time; physicians, emergency; quality assurance 